\title{
The EGFR Polymorphism Increased the Risk of Hepatocellular Carcinoma Through the miR-3196-Dependent Approach in Chinese Han Population
}

\section{Li Zhang \\ Xiaoping Li \\ Jiang Lu \\ Yi Qian \\ Tao Qian \\ Xing Wu \\ Qinghua Xu}

Department of Hepatobiliary and Pancreatic Surgery, LiYang People's Hospital, LiYang, 213300, Jiangsu, People's Republic of China
Correspondence: Qinghua Xu; Xing Wu Department of Hepatobiliary and Pancreatic Surgery, LiYang People's Hospital, 70\# JianShe West Road, LiYang, 213300, Jiangsu, People's Republic of China

$\mathrm{Tel} / \mathrm{Fax}+86-519-68091108$

Email xqh7888882@I63.com; sxj6628@I26.com
Background: Previous studies have shown that epidermal growth factor receptor (EGFR) promotes cell proliferation through the PI3K-Akt-mTOR signaling pathway and participates in the occurrence and development of hepatocellular carcinoma (HCC). Here, we focused on the functional polymorphism of EGFR in the 3 '-untranslated region (UTR), aiming to reveal the potential mechanisms by which functional polymorphism is associated with the risk and development of HCC in the Han Chinese population.

Methods: This study was a hospital-based case-control study. A total of 600 patients were enrolled, and another 600 healthy volunteers served as controls. The miR-associated SNPs in EGFR were screened, and genotyping was performed by TaqMan allele differential analysis. In this study, genotyping, real-time PCR, cell transfection and double luciferase reporter gene were used for subsequent analysis.

Results: HBV/HCV infection instead of alcohol exposure, smoking exposure, hypertension or diabetes mellitus was associated with an increased risk of HCC. Compared with TT genotypes, TG and GG genotypes of EGFR rs 884225 were significantly associated with reduced HCC risk. The stratified analysis of association between rs884225 and HCC subgroup feature reveal a highly correlation with tumor size. Furthermore, qRT-PCR confirmed that EGFR rs884225, TG and GG genotypes were more likely to bind to miR-3196 and down-regulate EGFR level in cells, thereby inhibiting cell proliferation.

Conclusion: This study suggested that EGFR rs 884225 is associated with a reduced risk of liver cancer and may be a developing biomarker.

Keywords: miR-3196, polymorphism, proliferation, hepatocellular carcinoma, HCC, epidermal growth factor receptor, EGFR

\section{Introduction}

Hepatocellular carcinoma (HCC) is one of the most common subtypes of liver cancer and one of the most common and deadliest cancers in the world. ${ }^{1}$ HCC is characterized by delayed diagnosis and poor prognosis. At present, targeted therapies for liver cancer have gradually advanced from sorafenib, a small molecule inhibitor, to PD-1-based immunotherapy., ${ }^{2,3}$ In China, most patients with liver cancer have a background of HBV or HCV infection. ${ }^{4,5}$ Studies have shown that genetics plays an important role in the occurrence and development of HCC, including single nucleotide polymorphisms (SNPs) and mutation time, and in 
addition, abnormal expression of protein-coding genes has also been confirmed to be involved in the development of HCC; ${ }^{6,7}$ However, the study of SNP in the $3^{\prime}$-untranslated region (UTR) of genes that are relatively key to the occurrence and development of HCC is still relatively rare.

Non-coding RNA (non-coding RNA) mainly includes miRNA (miRNA), long non-coding RNA (long non-coding RNA) and circular RNA (circRNA). Non-coding RNAs have been confirmed to play an important role in the occurrence and development of HCC, and may be involved in the interaction with protein-coding genes, leading to abnormal cell proliferation, invasion, differentiation, cell cycle change or apoptosis. ${ }^{8-11}$ Current studies have shown that miRNA plays a negative regulatory role on target genes mainly through the combination of complementary pairing with the $3^{\prime}$-UTR of downstream target genes. ${ }^{12,13}$ Current studies on genetic variation of HCC mainly focus on genotype changes in the exon regions of coding genes. Increasing evidence has proved that the miRNAs can participate in a polymorphism of gene transcription regulation, combined with the role of miRNAs main area for non-coding region, we put forward the idea, miRNAs may or with polymorphism in key genes that have different combination of ability, can alter the expression levels of genes and finally involved in disease development. ${ }^{14}$

Epidermal growth factor receptor (EGFR) is a tyrosine kinase transmembrane receptor, a member of the ERB family of receptors, mainly expressed on the surface of epithelial cells. ${ }^{15}$ EGFR has been shown to be involved in the regulation of multiple processes of tumorigenesis, including timing of cell survival, cell cycle progression, tumor invasion, and angiogenesis. ${ }^{16}$ Members of the EGFR family are involved in many cellular processes, such as cell proliferation and apoptosis, and play a central role in the development and progression of different types of cancer. ${ }^{17,18}$ In particular, abnormal changes in EGFR signaling have been identified as a key driver in the development of glioblastoma. In addition, in HCC, researchers have paid attention to SNPs in EGFR itself, which is closely related to the risk of disease occurrence, ${ }^{19}$ functional SNP located in the $3^{\prime} \mathrm{UTR}$ region of EGFR and the interaction with certain miRNA was poor investigated.

In this study, we first based on a public database miRNASNP (http://bioinfo.life.hust.edu.cn/miRNASNP/\#!)) for EGFR may interact with miRNA in SNP is forecasted, finally we focus on EGFR 3 'UTR of SNP (EGFR rs884225) site. Further validation was carried out with large samples to explore whether this SNP in EGFR was related to the occurrence of $\mathrm{HCC}$, or whether it caused different binding to miRNA and thus affected the expression and signal activation of EGFR.

\section{Materials and Methods \\ Clinical Samples}

Six-hundred patients with a clinicopathologic diagnosis of HCC and an equal number of healthy controls were included in the study. All blood samples are taken from Liyang People's Hospital during March 2009 to August 2019. Informed consent of patients was obtained before surgery, and relevant informed consent was signed. Blood samples were collected before operation and centrifuged within half an hour and frozen at $-80^{\circ} \mathrm{C}$. All peripheral blood samples were collected before the patient received any treatment. All patients in the control group were excluded from any other malignancy, genetic disease or autoimmune disease. This study has been approved by Liyang People's Hospital System Review Committee. All studies were carried out in accordance with government policy and the Helsinki Declaration.

\section{Genotyping and Real Time Polymerase Chain Reaction (RT-PCR)}

Genomic DNA was extracted from peripheral blood leukocytes using QIAGEN kit. The Quantitative RT-PCR were employed to detect the expression of target in this study. Total RNA was extracted from plasma samples and tissue samples by Trizol method. We also used TaqMan method to detect EGFR expression. U6 was used as endogenous reference during the whole detection process. Detection primer as follows: 5'-AGGCACGAGTAACAAGCTCAC-3' and 5' ATGAGGACATAACCAGCCAC-3 '. All genotypes were tested using the TaqMan probe and polymerase chain reaction (PCR) assay using a real-time PCR instrument according to ABI 7900HT company instructions.

\section{Dual Luciferase Reporter Gene Assay}

Firstly, the wild type of the 3 'UTR region of EGFR and the corresponding mutants at the binding site were cloned into the pGL4 promoter vector, respectively. The vectors obtained above, and miR-3196 mimics were transfected into HCC cell lines by the transfection method. In the transfection process, the Renal grass luciferase vector PRL-SV40 (5 ng) was transfected into the cell line as a reference, which was used as the background to test the standardized transfection 
efficiency. Fluorescence intensity in each group was detected by chemiluminescence analyzer for data analysis.

\section{CCK8 Assay}

CCK8 cell proliferation assay was used to detect the proliferation ability of HCC cell lines. Cells transfected with miR-3196 mimics of different genotypes or EGFRcontrols were inoculated on 96-well plates at a density of $1 \times 10^{4}$ cells/well and cultured for $48 \mathrm{~h}$. The absorbance values measured at $450 \mathrm{~nm}$ represent the DNA synthesis rate for data analysis. All CCK8 experiments were carried out with three replicates and three independent replicates.

\section{Statistical Analysis}

In the absence of special metasomatism, all data in this study are expressed as mean \pm standard deviation. Bilateral student $t$ test was used to compare the differences between the two groups. SPSS10.0 was used for all statistical analyses. $\mathrm{P}<0.05$ was considered statistically significant.

\section{Results}

\section{Characteristics of Participants}

According to the clinical information analysis of the patients included in this study, both the age and gender was matched between the control group and case group. The virus infection including $\mathrm{HBV} / \mathrm{HCV}$ were significantly associated with HCC. However, alcohol exposure, smoking exposure, hypertension or diabetes mellitus presented without significant difference (Table 1).

\section{Candidate Function SNP Located in the 3'UTR of EGFR}

In this study, we focused on the relationship between SNPs in EGFR 3'-UTR and HCC risk. We first in GenBank of SNP database (https://www.ncbi.nlm.nih.gov/snp) to retrieve the EGFR all 3'-the SNP UTR region, and through the other two conditions for further filtering: 1) Satisfy the MAF interval (0.05-0.1); and 2) Validation Status by- 1000 Genomes must be satisfied. Thus, potential SNP targets of EGFR function were obtained. And on this basis, according to the SNP further using bioinformatics software (http://bioinfo.life.hust.edu.cn/miRNASNP/\#!/) predict functional miRNA may interact with EGFR. According to the rank score provided by miRNASNP database, we focused on the functional SNP rs 884225 with the highest rank score. We further evaluated the
Table I Clinical Characteristics of HCC Patients and CancerFree Controls

\begin{tabular}{|c|c|c|c|c|c|}
\hline \multirow[t]{2}{*}{ Variables } & \multicolumn{2}{|c|}{$\begin{array}{c}\text { Cases } \\
(n=600)\end{array}$} & \multicolumn{2}{|c|}{$\begin{array}{l}\text { Controls } \\
(n=600)\end{array}$} & \multirow[t]{2}{*}{$P^{*}$} \\
\hline & $\mathbf{N}$ & $\%$ & $\mathbf{N}$ & $\%$ & \\
\hline \multicolumn{6}{|l|}{ Age (years) } \\
\hline$\leq 60$ & 350 & 58.3 & 330 & 55.0 & 0.244 \\
\hline$>60$ & 250 & 41.7 & 270 & 45.0 & \\
\hline \multicolumn{6}{|l|}{ Gender } \\
\hline Male & 481 & 80.2 & 482 & 80.3 & 0.942 \\
\hline Female & 119 & 19.8 & 118 & 19.7 & \\
\hline \multicolumn{6}{|l|}{ Virus infection } \\
\hline $\mathrm{HBV} / \mathrm{HCV}$ & 477 & 79.5 & 35 & 5.8 & $<0.000$ \\
\hline Negative & 123 & 20.5 & 565 & 94.2 & \\
\hline \multicolumn{6}{|l|}{ Alcohol exposure } \\
\hline Positive & 301 & 50.2 & 289 & 48.2 & 0.488 \\
\hline Negative & 299 & 49.8 & 311 & 51.8 & \\
\hline \multicolumn{6}{|l|}{ Smoking exposure } \\
\hline Positive & 302 & 50.3 & 291 & 48.5 & 0.525 \\
\hline Negative & 298 & 49.7 & 309 & 51.5 & \\
\hline \multicolumn{6}{|l|}{ Hypertension } \\
\hline Positive & 311 & 51.8 & 299 & 49.8 & 0.488 \\
\hline Negative & 289 & 48.2 & 301 & 50.2 & \\
\hline \multicolumn{6}{|l|}{ Diabetes mellitus } \\
\hline Positive & 219 & 36.5 & 203 & 33.8 & 0.333 \\
\hline Negative & 381 & 63.5 & 397 & 66.2 & \\
\hline
\end{tabular}

Notes: *Two-sided chi-square test for analyzing the clinical characteristics of HCC patients and cancer-free controls.

genotype frequency distribution of SNP rs884225 in 600 HCC patients and 600 healthy controls. Chi-square test confirmed that rs884225 genotype showed HardyWeinberg balanced distribution in healthy control group $(\mathrm{P}=0.41)$. As presented in Table 2, logistic regression analysis showed that TG and GG genotypes of EGFR rs 884225 significantly reduced the risk of HCC compared with TT genotypes (OR: 0.45; 95\% CI: TG genotype $0.21-0.66$, OR: 0.38 ; $95 \%$ CI: GG genotype was $0.33-$ 0.58); A higher number of $\mathrm{G}$ alleles was also associated with a reduced risk of HCC (OR:0.41;95\% confidence interval:0.25-0.61).

\section{Hierarchical Analysis of Correlation Between rs884225 and HCC Subgroup Features}

Next, we assessed the association of the EGFR rs884225 SNP with HCC subgroup feature. Patients with TG or GG 
Table 2 The Genotype Distribution of the SNP rs884225 in HCC Patients and Cancer-Free Controls

\begin{tabular}{|c|c|c|c|c|c|c|}
\hline \multirow[t]{2}{*}{ Genotype } & \multicolumn{2}{|c|}{ Cases $(n=600)$} & \multicolumn{2}{|c|}{ Controls $(n=600)$} & \multirow[t]{2}{*}{ OR $(95 \% \mathrm{Cl})^{\mathrm{a}}$} & \multirow[t]{2}{*}{$P$ value $^{\mathrm{a}}$} \\
\hline & $\mathbf{N}$ & $\%$ & $\mathbf{N}$ & $\%$ & & \\
\hline TT & 565 & 94.2 & 479 & 79.8 & 1.00 & 0.002 \\
\hline TG & 21 & 3.5 & 71 & 11.8 & $0.45(0.2 \mathrm{I}-0.66)$ & \\
\hline GG & 14 & 2.3 & 50 & 8.4 & $0.38(0.33-0.58)$ & \\
\hline G carrier & 35 & 5.8 & $12 \mid$ & 20.2 & $0.4 \mathrm{I}(0.25-0.6 \mathrm{I})$ & 0.01 \\
\hline
\end{tabular}

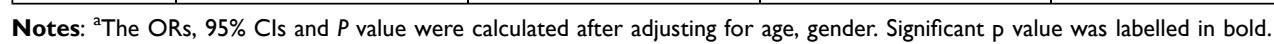

genotype presented a lower tumor size comparing with HCC patients with TT genotype. No statistical difference was obtained in tumor differentiation grade, tumor number, tumor capsular or metastasis (Table 3).

\section{EGFR rs884225 SNP Alteration of miR-3 196 Binding}

Thus far, we revealed that the EGFR rs884225 SNP was associated with risk and tumor size of HCC. According to the bioinformatics analysis, miR-3196 indicated the binding with MTHFR. Firstly, we detected the mRNA expression of EGFR in the HCC patients mentioned above, as presented in Figure 1A, we found a decreased level of
EGFR in patients with TG or GG genotype. CCK8 assay also revealed a decreased cell proliferation in these groups (Figure 1B). Bioinformatical analysis reveal that miR3196 might binding with the mutant type of EGFR (Figure 1C). We also found that patients with TT genotype presented a decreased level of miR-3196 comparing with TG or GG genotype (Figure 1D). The plasmid carried either the mutant type (GG genotype) or the wild type (TT genotype) was transfected into two HCC cells lines. We confirmed this binding by luciferase assay and found that the luciferase activity of the G-allele-specific PGL3 construct was significantly inhibited in the presence of miR-3196.In HepG2 and MHCC-97H cells, the luciferase

Table 3 The Stratified Analysis of Association Between rs884225 and HCC Risk

\begin{tabular}{|c|c|c|c|c|c|}
\hline \multirow[t]{2}{*}{ Feather } & \multicolumn{3}{|c|}{ Genotype } & \multirow{2}{*}{$\begin{array}{c}\text { TG vs TT } \\
P \text { value* }\end{array}$} & \multirow{2}{*}{$\begin{array}{c}\text { GG vs TT } \\
P \text { value* }\end{array}$} \\
\hline & TT & TG & GG & & \\
\hline \multicolumn{6}{|c|}{$\begin{array}{l}\text { Differentiation } \\
\text { grade }\end{array}$} \\
\hline Well & 301 & II & 6 & 0.782 & 0.726 \\
\hline Moderate & 156 & 7 & 5 & & \\
\hline Poorly & 108 & 3 & 3 & & \\
\hline \multicolumn{6}{|c|}{ Tumor number } \\
\hline Solitary & 344 & 15 & 9 & 0.330 & 0.797 \\
\hline Multiple & 221 & 6 & 5 & & \\
\hline \multicolumn{6}{|c|}{ Tumor size $(\mathrm{cm})$} \\
\hline$\leq 5 \mathrm{~cm}$ & 168 & 17 & 13 & $<0.001$ & 0.007 \\
\hline$>5 \mathrm{~cm}$ & 397 & 4 & I & & \\
\hline \multicolumn{6}{|c|}{ Tumor capsular } \\
\hline Incomplete & 182 & 7 & 5 & 0.914 & 0.782 \\
\hline Complete & 383 & 14 & 9 & & \\
\hline \multicolumn{6}{|l|}{ Metastasis } \\
\hline Yes & 188 & 7 & 4 & 0.996 & 0.712 \\
\hline No & 377 & 14 & 10 & & \\
\hline
\end{tabular}

Notes: *Two-sided chi-square test for either genotype distributions or allele frequencies between cases and controls. Significant $\mathrm{p}$ value was labelled in bold. 
A

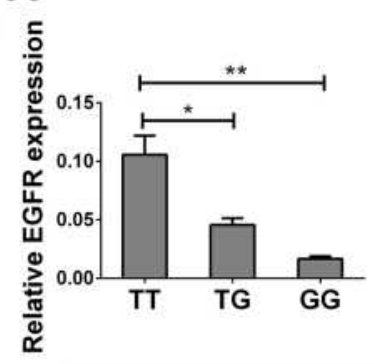

C

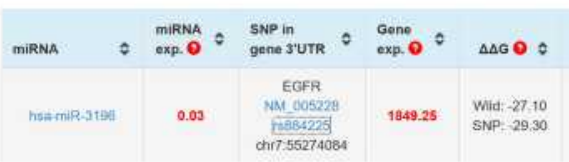

$E$

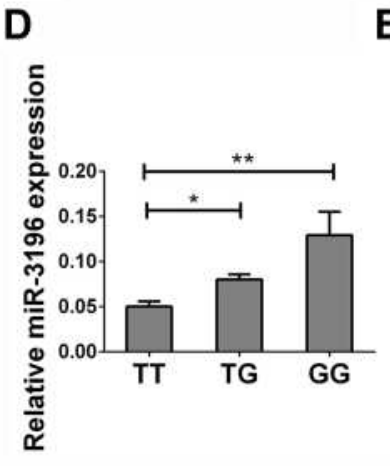

B

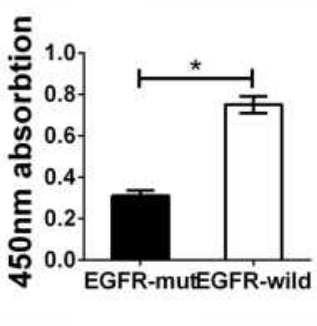

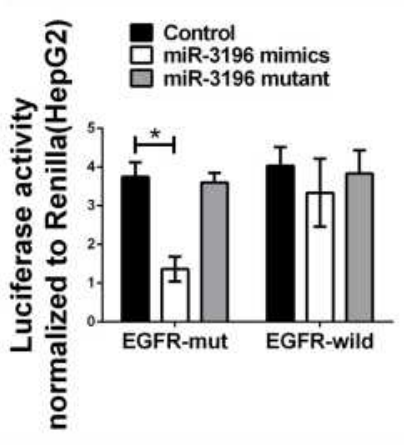

- LDSNP O EHoct

$\times$

gain

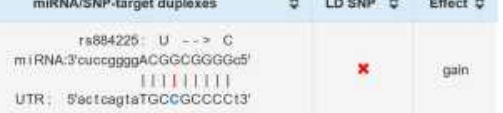

UTR: 5actcagtaTGCCGCCCC13

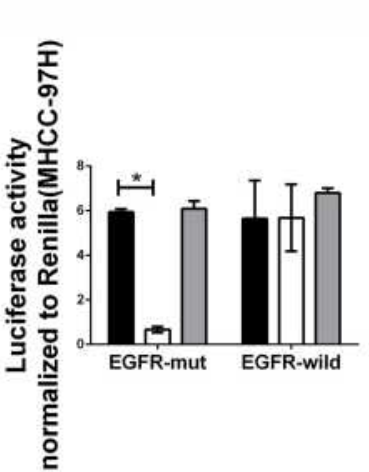

Figure I EGFR rs884225 SNP could bind with miR-3196 causing the suppression of EGFR and cell proliferation. (A) Expression of EGFR in HCC patients with TT, TG or GG genotype. (B) The CCK8 assay in HepG2 cell line treated with EGFR with different genotypes under miR-3196 stimulation. (C) Schematic diagram of specific binding sites of miRNA-3196 and EGFR. (D) Expression of miR-3196 in HCC patients with TT, TG or GG genotype. (E) Dual luciferase reporter gene assay in HepG2 and MHCC$97 \mathrm{H}$ cell lines. The data were presented as the mean \pm SD. $* *$ Indicates $P<0.01$. *Indicates $P<0.05$. (left panel) HepG2 cell line, (right panel) $M H C C-97 \mathrm{H}$ cell line.

activity of the A allele-specific PGL3 construct did not change after transfection with miR-3196, besides, the mutant type of miR-3196 presented no binding ability on EGFR (Figure 1E).

Based on the stratified analysis of association between rs884225 and patients' characteristics, we found that rs884225 had correlation with tumor size, and no correlation with tumor differentiation grade, tumor number, tumor capsular and metastasis. Thus, according to your suggestion, we thought it is necessary to detect the function of miR-3196 on HCC cell's proliferation and the expression of EGFR related factors including Ki67 and phosphorylation of ERK/AKT. We found that overexpression of miR-3196 will reduced the cell proliferation only when cells was overexpressed with EGFR mutant type, the expression of Ki67 was also decreased with the suppression of ERK/AKT phosphorylation (Figure 2A and B).

\section{Discussion}

In this study, we found that polymorphism at EGFR (rs884225) suggested a lower risk of HCC. Further functional analysis suggested that this polymorphic locus and miRNA might interact with each other, enabling miR-3196 to have a stronger affinity with EGFR, thus binding to the 3'UTR region of EGFR, thereby inhibiting EGFR expression level and further inhibiting cell proliferation.

EGFR is a known transmembrane tyrosine kinase receptor. Existing studies have shown that the function of EGFR is mainly involved in the signal transduction of DNA repair, tumor cell survival and cell proliferation, and plays its role through a variety of signal pathways such as PI3K-PTEN-Akt and Ras-RAF-MAPK signaling cascades. $^{20}$ Genetic variation of EGFR gene is also a research hotspot at present. Studies have confirmed that EGFR specific mutation exists in most cancer patients, and 
A

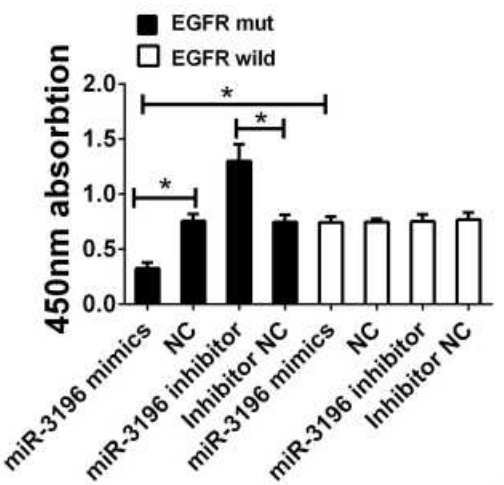

B

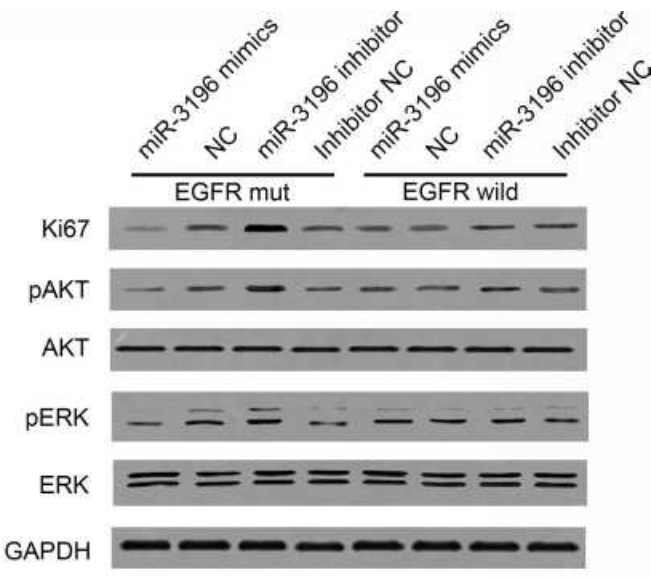

Figure 2 EGFR rs884225 SNP reduced the cell proliferation through the miR-3196-dependent approach. (A) CCK8 assay was detected in HCC cells treated with different expression of miR-3196 with EGFR mutant type or wild type. (B) The expression of Ki67, phosphorylation of ERK and AKT in HCC cells. *Indicates P<0.05.

this type of mutation is closely related to DNA amplification and allele copy number variation..$^{21,22}$

Through a searchable database, we found that in The Cancer Genome Atlas (TCGA) in the league has included information about EGFR somatic mutations, in human tumors, six mutation of EGFR have been proved in human cancers, their site distributed throughout the whole structure of EGFR gene mutation, both including the extracellular and intracellular domain of EGFR, indicating that EGFR mutations may be involved in the process of the whole signal to include with the combination of the ligands and the downstream signal transduction. ${ }^{23,24} \mathrm{In}$ the study of HCC, we have found that abnormal activation of EGF/EGFR signaling pathway is one of the key factors in the development of HCC. ${ }^{25}$ In accordance with this, in the studies on the occurrence and development of EGFR polymorphism in liver cancer, rs4947986 polymorphism was highly correlated with the susceptibility to liver cancer. The study found that rs4947986 polymorphism was located 47 base from the boundary of exon 7, which was the key site for the dimerization of EGFR. ${ }^{26}$

Among the studies on the polymorphism related effects of miRNAs with specific target genes, some researchers have found that some miRNAs can bind to some polymorphic target genes $3^{\prime}$ UTR. For example, in irritable bowel syndrome, miR-16 and miR-103 have been proved to have a high affinity with the specific receptor signals of 5-HT, affecting downstream signal transduction. ${ }^{27}$ In addition, similar reports have been reported in human cancer diseases. In prostate cancer patients, miR-502 can bind to the 3'UTR polymorphism of SETD8 gene and specifically inhibit the expression of SETD8, increasing the risk of prostate cancer. ${ }^{28}$ In gastrointestinal tumors, there are also relevant reports on functional SNPs, especially in the Han population. As for gastrointestinal tumors, in the Chinese Han population, the BSG gene $3^{\prime}$-UTR has functional polymorphic sites that can specifically bind to miR-483$5 p$, thus affecting the occurrence of esophageal cancer. ${ }^{29}$ In addition, in HCC, it has also been reported that miR214 regulates U/G SNP rs111904020 in STAT3 3'UTR, which promotes the development of HCC in Chinese population. Similarly, FOXO has similar functional SNPs that bind to specific miRNAs and are highly associated with the risk of oncogenesis. ${ }^{30}$

\section{Conclusions}

To sum up, this study found closely related to liver cancer of EGFR gene 3 'UTR region exists specificity of polymorphism loci is closely related to the occurrence of liver cancer risk, at the same time, the polymorphism and miR-3196 has a relatively strong affinity, and then under the function of miR-3196 inhibits the expression of EGFR, at the same time reduce the proliferation of tumor cells. Of course, this study also has some defects, because it is a hospital-based casecontrol study with certain selection bias, and large-scale verification in a larger sample is needed in the later stage.

\section{Disclosure}

The authors declare that they have no conflicts of interest. 


\section{References}

1. Rajyaguru DJ, Borgert AJ, Smith AL, et al. Radiofrequency ablation versus stereotactic body radiotherapy for localized hepatocellular carcinoma in nonsurgically managed patients: analysis of the National Cancer Database. J Clin Oncol. 2018:JCO2017753228.

2. Loo TM, Kamachi F, Watanabe Y, et al. Gut microbiota promotes obesity-associated liver cancer through PGE2-mediated suppression of antitumor immunity. Cancer Discov. 2017;7(5):522-538. doi:10.1158/2159-8290.CD-16-0932

3. Cast A, Valanejad L, Wright $\mathrm{M}$, et al. C/EBPalpha-dependent pre-neoplastic tumor foci are the origin of hepatocellular carcinoma and aggressive pediatric liver cancer. Hepatology. 2017.

4. Yan J, Caviglia JM, Schwabe RF. Animal models of HCC - When injury meets mutation. J Hepatol. 2017. doi:10.1016/j.jhep.2017.07.023

5. Li J, Wang X, Tang J, et al. HULC and Linc00152 act as novel biomarkers in predicting diagnosis of hepatocellular carcinoma. Cell Physiol Biochem. 2015;37(2):687-696. doi:10.1159/000430387

6. Huang DH, Wang GY, Zhang JW, Li Y, Zeng XC, Jiang N. MiR-501$5 p$ regulates CYLD expression and promotes cell proliferation in human hepatocellular carcinoma. Jpn J Clin Oncol. 2015;45 (8):738-744. doi:10.1093/jjco/hyv063

7. Miki D, Ochi H, Hayes CN, et al. Variation in the DEPDC5 locus is associated with progression to hepatocellular carcinoma in chronic hepatitis C virus carriers. Nat Genet. 2011;43(8):797-800. doi:10.1038/ng.876

8. Wang R, Zhang J, Ma Y, et al. Association study of miR149 rs2292832 and miR608 rs4919510 and the risk of hepatocellular carcinoma in a largescale population. Mol Med Rep. 2014;10 (5):2736-2744. doi:10.3892/mmr.2014.2536

9. Jiang R, Tang J, Chen Y, et al. The long noncoding RNA lnc-EGFR stimulates T-regulatory cells differentiation thus promoting hepatocellular carcinoma immune evasion. Nat Commun. 2017;8(1):15129. doi: $10.1038 /$ ncomms 15129

10. Tang J, Zhuo H, Zhang X, et al. A novel biomarker Linc00974 interacting with KRT19 promotes proliferation and metastasis in hepatocellular carcinoma. Cell Death Dis. 2014;5(12):e1549. doi:10.1038/cddis.2014.518

11. Chang Y, Yan W, He X, et al. miR-375 inhibits autophagy and reduces viability of hepatocellular carcinoma cells under hypoxic conditions. Gastroenterology. 2012;143(1):177-187 e178. doi:10.1053/j.gastro.2012.04.009

12. Panganiban RP, Wang Y, Howrylak J, et al. Circulating microRNAs as biomarkers in patients with allergic rhinitis and asthma. J Allergy Clin Immunol. 2016;137(5):1423-1432. doi:10.1016/j. jaci.2016.01.029

13. Zhou X, Ye F, Yin C, Zhuang Y, Yue G, Zhang G. The interaction between MiR-141 and lncRNA-H19 in regulating cell proliferation and migration in gastric cancer. Cell Physiol Biochem. 2015;36 (4):1440-1452. doi:10.1159/000430309

14. Chen Q, Qin R, Fang Y, Li H, Liu Y, Functional A. Variant at the miR-214 binding site in the methylenetetrahydrofolatereductase gene alters susceptibility to gastric cancer in a Chinese Han Population. Cell Physiol Biochem. 2015;36(2):622-630. doi:10.1159/000430125

15. Bosch A, Eroles P, Zaragoza R, Vina JR, Lluch A. Triple-negative breast cancer: molecular features, pathogenesis, treatment and current lines of research. Cancer Treat Rev. 2010;36(3):206-215. doi:10.1016/j.ctrv.2009.12.002
16. Panvichian R, Tantiwetrueangdet A, Sornmayura P, Leelaudomlipi S. Missense mutations in Exons 18-24 of EGFR in hepatocellular carcinoma tissues. Biomed Res Int. 2015;2015:171845. doi:10.1155/ 2015/171845

17. Xu S, Duan Y, Lou L, Tang F, Shou J, Wang G. Exploring the impact of EGFR T790M neighboring SNPs on ARMS-based T790M mutation assay. Oncol Lett. 2016;12(5):4238-4244. doi:10.3892/ ol.2016.5184

18. Charoen P, Nitsch D, Engmann J, et al. Mendelian Randomisation study of the influence of eGFR on coronary heart disease. Sci Rep. 2016;6(1):28514. doi:10.1038/srep28514

19. Wang W, Ma XP, Shi Z, et al. Epidermal growth factor receptor pathway polymorphisms and the prognosis of hepatocellular carcinoma. Am J Cancer Res. 2015;5(1):396-410.

20. Ghasimi S, Wibom C, Dahlin AM, et al. Genetic risk variants in the CDKN2A/B, RTEL1 and EGFR genes are associated with somatic biomarkers in glioma. $J$ Neurooncol. 2016;127(3):483-492. doi:10.1007/s11060-016-2066-4

21. You KS, Yi YW, Kwak SJ, Seong YS. Inhibition of RPTOR overcomes resistance to EGFR inhibition in triple-negative breast cancer cells. Int J Oncol. 2018. doi:10.3892/ijo.2018.4244

22. Li B, Ding CM, Li YX, Peng JC, Geng N, Qin WW. Over-regulation of microRNA-133b inhibits cell proliferation of cisplatin-induced non-small cell lung cancer cells through PI3K/Akt and JAK2/ STAT3 signaling pathway by targeting EGFR. Oncol Rep. 2018.

23. Iderzorig T, Kellen J, Osude C, et al. Comparison of epithelial mesenchymal transition mediated tyrosine kinase inhibitor resistance in non-small cell lung cancer cell lines with wild type EGFR and mutant type EGFR. Biochem Biophys Res Commun. 2018;496 (2):770-777. doi:10.1016/j.bbrc.2018.01.069

24. Tang Q, Wang Y, Ma L, et al. Peiminine serves as an adriamycin chemosensitizer in gastric cancer by modulating the EGFR/FAK pathway. Oncol Rep. 2018. doi:10.3892/or.2018.6184

25. Thompson SM, Jondal DE, Butters KA, et al. Heat stress induced, ligand-independent MET and EGFR signalling in hepatocellular carcinoma. Int J Hyperthermia. 2017:1-12.

26. Girard N. Optimizing outcomes in EGFR mutation-positive NSCLC: which tyrosine kinase inhibitor and when? Future Oncol. 2018. doi:10.2217/fon-2017-0636

27. Wohlfarth C, Schmitteckert S, Hartle JD, et al. miR-16 and miR-103 impact 5-HT4 receptor signalling and correlate with symptom profile in irritable bowel syndrome. Sci Rep. 2017;7(1):14680. doi:10.1038/ s41598-017-13982-0

28. Narouie B, Ziaee SAM, Basiri A, Hashemi M. Functional polymorphism at the miR-502-binding site in the $3^{\prime}$ untranslated region of the SETD8 gene increased the risk of prostate cancer in a sample of Iranian population. Gene. 2017;626:354-357. doi:10.1016/j. gene.2017.05.060

29. Li HY, Liu YC, Bai YH, et al. SNP at miR-483-5p-binding site in the 3'-untranslated region of the BSG gene is associated with susceptibility to esophageal cancer in a Chinese population. Genet Mol Res. 2016;15(2).

30. Fan Y, Qian X, Zhang C. U/G SNP rs111904020 in 3'UTR of STAT3 regulated by miR-214 promotes hepatocellular carcinoma development in Chinese population. Tumour Biol. 2016;37(11):14629-14635. doi:10.1007/s13277-016-5352-z 


\section{Publish your work in this journal}

Pharmacogenomics and Personalized Medicine is an international, peer-reviewed, open access journal characterizing the influence of genotype on pharmacology leading to the development of personalized treatment programs and individualized drug selection for improved safety, efficacy and sustainability. This journal is indexed on the American Chemical Society's Chemical Abstracts Service (CAS). The manuscript management system is completely online and includes a very quick and fair peer-review system, which is all easy to use. Visit http://www.dovepress.com/testimonials.php to read real quotes from published authors. 